\title{
Retraction Note to: Context-Aware Location Recommendations for Smart Cities
}

\author{
Akanksha Pal and Abhishek Singh Rathore
}

\section{Retraction Note to: \\ Chapter "Context-Aware Location Recommendations \\ for Smart Cities" in: F. Al-Turjman (ed.), \\ Smart Cities Performability, Cognition, \& Security, EAI/Springer Innovations in Communication and Computing, https://doi.org/10.1007/978-3-030-14718-1_5}

The editor has retracted this chapter [1] because a significant part of the text and Fig. 5.3 overlap with a previously published conference paper by Wagih et al. [2]. Akanksha Pal agrees with this retraction. Abhishek Singh Rathore has not responded to correspondence about this retraction.

[1] Pal, A., \& Rathore, A. S. (2020). Context-aware location recommendations for smart cities. In: F. Al-Turjman (Ed.), Smart cities performability, cognition, \& security. EAI/Springer Innovations in Communication and Computing. Cham: Springer.

[2] Wagih, H., Mokhtar, H., \& Ghoniemy, S. (2017). Location recommendation based on social trust. In: Thirteenth international conference on semantics, knowledge and grids (SKG), Beijing, pp. 50-55. https://doi.org/10.1109/SKG.2017.00017

The retracted version of this chapter can be found at https://doi.org/10.1007/978-3-030-14718-1_5 